\title{
CALIDAD DEL ARROZ DE TIERRAS ALTAS EN FUNCIÓN DEL TIEMPO DE COCCIÓN Y DEL CULTIVAR DE ARROZ
}

\section{RICE QUALITY EVALUATION AS A FUNCTION OF CULTIVAR AND COOKING TIME}

\author{
Diego Palmiro Ramirez ASCHERI ${ }^{1}$ \\ Luciane Dias PEREIRA ${ }^{2}$ \\ Priscila Zaczuk BASSINELLO ${ }^{3}$ \\ Carlos Wanderlei Piler CARVALHO \\ José Luís Ramírez ASCHERI ${ }^{5}$
}

\begin{abstract}
RESUMEN
Se han estudiado los efectos que causan el cultivar y el tiempo de cocción en la calidad de granos de cinco cultivares de arroz de tierras altas (BRS Primavera, BRS Pepita, BRS Sertaneja, BRS MG Curinga y BRS Monarca) que fueron cocidos en diferentes tiempos de cocción (0,10,20,30 e $40 \mathrm{~min}$ ). Se han determinado los contenidos de agua, almidón y amilosa, viscosidad máxima y tendencia a la retrogradación y se realizaron ensayos de cocción (rendimiento, pegajosidad y textura). Los resultados demostraron que cuanto mayor es el tiempo de cocción mayor es la disponibilidad de los contenidos de almidón y de amilosa para sus determinaciones y sus valores difieren entre los cultivares de arroz de tierras altas. Se ha observado sinergismo entre los factores estudiados, aumentando el contenido de agua y disminuyendo la viscosidad máxima y la tendencia a la retrogradación con el aumento del tiempo de cocción se ha notado que los cultivares BRS Pepita y BRS MG Curinga pueden absorber mayor cantidad de agua cuando calientes y presentarse más pegajosos cuando fríos y los cultivares BRS Primavera, BRS Sertaneja y BRS Monarca pueden absorber menor cantidad de agua cuando calientes y sus granos pueden presentarse secos y sueltos y endurecerse después de enfriados.

Palabras-clave: Oryza sativa; tecnología de cereales; viscosidad de pasta; almidón.
\end{abstract}

\begin{abstract}
Was studied the effects caused by the cultivar and cooking time on grain quality of five cultivars of upland rice (BRS Primavera, BRS Pepita, BRS Sertaneja, BRS Monarca and BRS MG Curinga) that were cooked at different cooking times ( 0 , $10,20,30$ and $40 \mathrm{~min}$ ). The identified water content, starch and amylose, high viscosity and tendency to retrogradation and cooking tests were performed (yield, stickiness and texture). The results showed that the longer the cooking time the greater the availability of the contents of starch and amylose for their determinations and their values differ among cultivars of upland rice. Synergism was observed between the factors studied, increasing the water content and decreasing the maximum viscosity and the tendency to retrogradation with increased cooking time has been noted that cultivars BRS Pepita and BRS MG Curinga can absorb more water when they are hot and presented more stickiness when is cold and cultivars BRS Primavera, BRS Sertaneja and BRS Monarca can absorb minor water when they are hot and can be show dry grains and singles and harden after cooling.
\end{abstract}

Key-words: Oryza sativa; cereals technology; pasting viscosity; starch.

\footnotetext{
${ }^{1}$ Ingeniero de Alimentos, Dr. Ms. Sc. Prof. de la Universidad Estatal de Goiás. Rua 10, Quadra 25, Lote 20, Bairro São João, CEP: 75.133-130, Anápolis, Goiás, Brazil. E-mail: ascheridpr@uol.com. Autor para correspondencia.

${ }^{2}$ Bachiller en Química Industrial de la Universidad Estatal de Goiás. Anápolis, Goiás, Brazil. E-mail: luciane dpereira@hotmail.com.

3 Eng. Agrónoma., Dra. Sc. Investigadora Científico - Embrapa Arroz e Feijão. Rodovia GO-462, Km 12. Zona Rural 75375-000 - Santo Antonio de Goiás, Goiás, Brazil. E-mail: pzbassin@cnpaf.embrapa.br.

${ }^{4}$ Ingeniero Agrônomo, Dr. Sc. Investigador Científico - Embrapa Agroindústria de Alimentos. Av. de las Américas 29501, Guaratiba. CEP 23020-470, Rio de Janeiro, Rio de Janeiro, Brazil. E-mail: cwpiler@ctaa.embrapa.br.

${ }^{5}$ Ingeniero de Alimentos, Dr. Sc. Investigador Científico - Embrapa Agroindústria de Alimentos. Rio de Janeiro, Rio de Janeiro, Brazil. E-mail: ascheri@ctaa.embrapa.br
} 
ASCHERI , D.P.R. et al. Calidad del arroz de tierras altas...

\section{INTRODUCCIÓN}

El arroz constituye uno de los cereales básicos de la dieta humana, representando aproximadamente $20 \%$ de la ingestión mundial de energía y $15 \%$ del aporte de proteína. En los países más pobres del Asia, el consumo de arroz corresponde más de la mitad del aporte energético y proteico de esas poblaciones (Kennedy \& Burlingame, 2003).

Dada la gran importancia económica y nutricional del arroz y como mayor productor de este cereal en América Latina y décimo mayor productor mundial (FAO, 2004), el Brasil viene dedicándose a la investigación de nuevos cultivares de alta calidad de granos, precoces, resistentes a enfermedades, con elevado potencial productivo y de alta rentabilidad. Sin embargo, debido a la velocidad con que se está desarrollando estos cultivares aún hay necesidad de estudiar sus características físico-químicas que puedan evaluar con cierta facilidad la calidad del arroz.

El principal componente de la calidad del arroz es la textura que es determinada a través de una evaluación sensorial en que muchas veces se necesitan de personas entrenadas para describirlo. Sin embargo, resulta costoso para los programas de mejoramiento genético, disponer tamaño de muestra que se ajuste a la metodología exigida por las pruebas sensoriales. Por otra parte, los panelistas o jueces sensoriales pueden disminuir su precisión al ser sometidos a un alto número de sesiones (Ávila, 2001).

Una de las formas de evaluación de la calidad del arroz y la realización de ensayos para determinar la textura del arroz es el contenido de la amilosa en el almidón (Kennedy \& Burlingame, 2003; Ferreira et al., 2005). El contenido de amilosa determina las características de consistencia, viscosidad y pegajosidad del arroz durante y después de la cocción (Wang et al., 2000; Ávila, 2001; Scholz \& Magri, 2003). Las determinaciones de esas características se consideran métodos indirectos que evalúan la calidad cuantificando el comportamiento de las pastas de arroz frente a la cocción, lo cual está estrechamente relacionado con el grado de pegajosidad de los granos.

Entretanto, la literatura ha demostrado que cuando el arroz es sometido a diferentes procesos físico-químicos, este grano presenta diferentes calidades. Juliano (1985) demostró que en la cocción del arroz por medio de microondas, la cantidad de agua utilizada durante la cocción y el vapor pueden afectar la textura y el sabor del arroz. Pinkrová et al. (2003) observaron que el almidón dañado contenido en los granos de arroz aumenta con el aumento de la energía irradiada absorbida, concomitantemente con la concentración de agua e temperatura utilizada. Bao et al. (2005), Yu \& Wang (2007) y Zanão et al. (2009) observaron que las propiedades de pasta del arroz fueron significativamente alteradas, ocurriendo la reducción de la temperatura de pasta, disminución del tiempo para la ocurrencia del pico de viscosidad, reducción en los valores de viscosidad máxima y viscosidad final y la tendencia de retrogradación se tornó menos acentuada en granos con dosis creciente de radiación gamma.

El presente trabajo tubo como objetivo estudiar algunas propiedades físico-químicas de cinco cultivares de arroz de tierras altas sometidos a diferentes tiempos de cocción.

\section{MATERIAL Y MÉTODOS}

Las muestras de arroz se obtuvieron del campo experimental de la Embrapa Arroz e Feijão (Santo Antônio de Goiás/GO), sembradas en noviembre de 2006 y cosechadas en marzo de 2007. Las semillas, con aproximadamente 13\% de humedad en base seca, fueron descascaradas y pulidas en un molino experimental Suzuki, MT-95 (Suzuki Co., São Paulo, Brasil) con grado de molienda de $10 \%$.

Se utilizó un diseño experimental completamente casualizado con arreglo factorial de $5^{2}$ tratamientos: cinco cultivares (C) de arroz (BRS Primavera, BRS Pepita, BRS Monarca, BRS Sertaneja, BRS Curinga) y cinco tiempos ( $T$ ) de cocción [0, (testigo), 10, 20, 30 y $40 \mathrm{~min}$ ], con tres repeticiones. Las variables respuestas fueron: los contenidos de agua (Hu), almidón (Al) y amilosa $(A m)$ y la viscosidad obtenida por el Analizador Rápido de Viscosidad (RVA) del cual se obtuvo la viscosidad máxima (Vmáx) y la ocurrencia de la retrogradación $(\mathrm{Sb})$. Se realizó un análisis de varianza usando el programa Statistica 6.0 (Statsoft, 2007) y las medias de las variables cualitativas significativas se compararon entre sí usando la prueba de Tukey al nivel de $5 \%$ de probabilidad ( $p \leq$ $0.05)$. Se realizaron análisis de regresión para estimar las variables cuantitativas significativas. El criterio para elegir el modelo de regresión fue el que presentara menor cuadrado medio del error (MSE) al nivel de $5 \%$ de probabilidad (Gomes, 1987). La correlación ( $r$ ) entre los resultados se obtuvo con los valores medios de las variables entre 10 y $40 \mathrm{~min}$ de cocción.

Para la cocción del arroz se utilizó la metodología descripta por Bassinello et al. (2004) con modificaciones para el tiempo de cocimiento. Se adicionó en una olla el arroz y el agua y se dejó hervir hasta antes del agua secar, seguidamente se tapó la olla y se disminuyó el flujo del gas para continuar cocinando a fuego lento. Para eso se utilizó $150 \mathrm{~g}$ de arroz y $300 \mathrm{~cm}^{3}$ de agua destilada para cada tiempo de cocción establecido entre 10 y 40 min. Durante la cocción se adicionó más 75, 150 y $225 \mathrm{~cm}^{3}$ de agua para completar los tiempos de 20, 30 y 40 min de cocción, respectivamente, caso contrario, las muestras se quemaban. La muestras crudas y cocidas fueron deshidratadas en estufa Fanem 520 (Fanem, São Paulo, Brasil) a $45^{\circ} \mathrm{C}$ por $16 \mathrm{~h}$ y molidas en molino de cuchillas Perten Laboratory Mill 3100 (Perten instruments, Huddinge, Suecia), obteniéndose las harinas de arroz.

El contenido de agua fue determinado en base al método de la AMERICAN ASSOCIATION 
ASCHERI , D.P.R. et al. Calidad del arroz de tierras altas...

OF CEREAL CHEMISTS (1969), a $105^{\circ} \mathrm{C}$ hasta masa constante. El contenido de almidón se determinó utilizando el método de Lane-Enyon (Cereda et al., 2004), por medio de digestión ácida en microondas. El contenido de amilosa se evaluó por el método colorimétrico de Martinez \& Cuevas (1989), utilizando como indicador la solución yodo/ yoduro de potasio y el complejo formado medido por espectrofotómetro (Fento, mod. 432) a $620 \mathrm{~nm}$.

La viscosidad máxima (Vmáx) y la tendencia a la retrogradación (set back, $\mathrm{Sb}$ ) fueron obtenidas de los perfiles de viscosidad de pasta de los granos de arroz, determinados en RVA (Rapid Visco Analyzer 4, Newport Scientific PTY LTD, Sydney, Australia) (NEWPORT SCIENTIFIC, 1998), con algunas modificaciones, donde $3 \mathrm{~g}$ de harina (a $14 \%$ en base húmeda) cribado en malla de $250 \mu \mathrm{m}$ fueron suspendidos en agua destilada hasta completar $28 \mathrm{~g}$. El perfil de viscosidad fue diseñado de acuerdo con el siguiente régimen tiempo/ temperatura: $25^{\circ} \mathrm{C}$ por 2 min, calentamiento de 25 a $95^{\circ} \mathrm{C}$ a una tasa de $14{ }^{\circ} \mathrm{C} \mathrm{min}^{-1}$, mantención de la pasta a $95{ }^{\circ} \mathrm{C}$ por 3 min (ciclo de calentamiento), enfriamiento de 95 a $25^{\circ} \mathrm{C}$ a una tasa de $12{ }^{\circ} \mathrm{C} \mathrm{min}$ ${ }^{1}$ (ciclo de enfriamiento) y mantención de la pasta a $25^{\circ} \mathrm{C}$ por $4 \mathrm{~min}$. El valor de Vmáx fue detectado en el pico máximo de viscosidad formado durante el ciclo de calentamiento y el valor de $\mathrm{Sb}$ fue determinado por la diferencia del valor de la viscosidad final (detectado en el tiempo de $20 \mathrm{~min}$ de lectura del RVA) con el valor mínimo de viscosidad detectado durante el ciclo de enfriamiento.

El ensayo de cocción fue realizado para evaluar el rendimiento (\%), pegajosidad y textura de los granos cocidos. Los granos fueron cocidos simulando la preparación casera del consumidor (Bassinello et al., 2004), colocándose $300 \mathrm{~g}$ de arroz en $570 \mathrm{~cm}^{3}$ de agua, $2 \mathrm{~g}$ de sal y $60 \mathrm{~cm}^{3} \mathrm{de}$ aceite de soya.

El análisis de pegajosidad fue hecha inmediatamente después de la cocción de los granos de arroz en cuanto que el análisis de la textura fue realizado dejando los granos enfriar a la temperatura ambiente (aproximadamente $25{ }^{\circ} \mathrm{C}$ ) (Martinez \& Cuevas, 1989). Estos atributos fueron obtenidos usando cinco probadores entrenados de la EMBRAPA Arroz e Feijão y se utilizó el teste de escala estructurada en donde las muestras fueron presentadas a los probadores en cabinas individuales, en forma monódica, cuya orden de presentación fue definida por medio de sorteo. Se solicitó a los probadores que disgustasen las muestras y enjuagasen la boca después de cada degustación y anotasen la posición de la escala que mejor represente su prueba de la intensidad de textura y pegajosidad para cada muestra, de acuerdo con la evaluación de la Tabla 1.

TABLA 1 - Evaluación para escala estructurada (verbal y bipolar) de textura y pegajosidad de arroz.

\begin{tabular}{ll}
\hline \multicolumn{1}{c}{ Textura } & \multicolumn{1}{c}{ Pegajosidad } \\
\hline 1. Extremamente suave & 1. Extremamente suelto \\
2. Suave & 2. Muy suelto \\
3. Ligeramente suave & 3. Suelto \\
4. Suave con el centro firme & 4. Ligeramente suelto \\
5. Levemente firme & 5. Pegajoso \\
6. Muy firme & 6. Muy pegajoso \\
7. Extremamente firme & 7. Extremamente pegajoso \\
\hline
\end{tabular}

Fuente: Adaptado de Martinez \& Cuevas (1989).

El rendimiento (Equación 1) fue calculado de acuerdo con la cantidad de tazas ( $Q$ ) obtenidas de arroz cocido. Las tazas (de $200 \mathrm{~cm}^{3}$ de capacidad) fueron llenadas al ras con los granos cocidos de arroz con la ayuda de una cuchara.

$$
\text { Rendimiento }(\%)=\left[\frac{\mathrm{Q}}{2} \times 100\right]-100
$$

Se registró el tiempo de cocción de cada muestra de arroz analizada de manera no estandarizada, o sea, el tiempo fue leído así que el arroz "secó" en la olla, conforme es realizado en la culinaria diaria. 
ASCHERI , D.P.R. et al. Calidad del arroz de tierras altas...

\section{RESULTADOS Y DISCUSIÓN}

De acuerdo con la Tabla 2, la interacción C x $T$ fue significativa para el tenor de agua $(p<0.01)$; sin embargo, para almidón y amilosa no lo fue $(p>0.05)$, indicando que no existen evidencias suficientes para concluir que el efecto del factor tiempo de cocción en estas variables depende del efecto del factor cultivar de arroz de tierras altas.

TABLA 2 - Análisis de varianza de los contenidos de almidón (Al), amilosa (Am) y agua (Hu) de los cultivares de arroz de tierras altas, obtenidos en diferentes tiempos de cocción.

\begin{tabular}{|c|c|c|c|c|c|c|c|}
\hline \multirow{2}{*}{ Fuente de variación } & \multirow{2}{*}{ G.I. } & \multicolumn{3}{|c|}{ Cuadrado medio } & \multicolumn{3}{|c|}{ Prueba F } \\
\hline & & $\mathrm{Al}$ & $\mathrm{Am}$ & $\mathrm{Hu}$ & $\mathrm{Al}$ & Am & $\mathrm{Hu}$ \\
\hline Cultivar (C) & 4 & 30.6 & 12.9 & 0.36 & $30.7^{* \star *}$ & $87.0^{\star * \star}$ & $16456.1^{\star \star \star}$ \\
\hline Tiempo $(\mathrm{T})$ & 4 & 35.4 & 24.0 & 13.68 & $35.5^{\star \star \star}$ & $162.4^{\star \star *}$ & $618930.4^{\star \star \star}$ \\
\hline $\mathrm{C} \times \mathrm{T}$ & 16 & 0.6 & 0.2 & 0.05 & $0.6^{\mathrm{ns}}$ & $1.3^{\text {ns }}$ & $2212.9^{\star \star \star}$ \\
\hline
\end{tabular}

G.I. = grados de libertad; ${ }^{* \star *}$ Muy significativo $(p<0.001) ;{ }^{\text {ns }}$ No significativo $(p>0.05)$. - No determinado.

En las Tablas 3 y 4 se muestran, respectivamente, los tenores medios y errores estándar del almidón y de la amilosa. Las medias entre cultivares e entre tiempos de cocción difirieron entre sí por la prueba de Tukey $(p<0.05)$. El almidón y la amilosa entre cultivares variaron de 90.61 a
94.26 dag $\mathrm{kg}^{-1}$ y de 22.82 a 25.19 dag $\mathrm{kg}^{-1}$, respectivamente, no siendo diferente cuando sometidos a los diferentes tiempos de cocción,

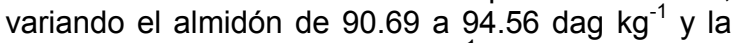
amilosa de 22.53 a 25.72 dag $\mathrm{kg}^{-1}$.

TABLA 3 - Contenido de almidón $\left(\right.$ dag $\mathrm{kg}^{-1}$ ) de cultivares de arroz de tierras altas sometidos a diferentes tiempos de cocción.

\begin{tabular}{|c|c|c|c|c|c|c|}
\hline Tempo & $0 \min$ & $10 \mathrm{~min}$ & $20 \min$ & $30 \mathrm{~min}$ & $40 \mathrm{~min}$ & DMS \\
\hline $\bar{x}$ & $90.69^{d}$ & $91.57^{\mathrm{cd}}$ & $92.48^{c}$ & $93.53^{b}$ & $94.56^{a}$ & 0,91 \\
\hline CV (\%) & 1.4 & 1.45 & 1.7 & 1.87 & 2.14 & - \\
\hline Cultivar & $\begin{array}{c}\text { BRS } \\
\text { Primavera }\end{array}$ & $\begin{array}{l}\text { BRS } \\
\text { Pepita }\end{array}$ & $\begin{array}{c}\text { BRS } \\
\text { Sertaneja }\end{array}$ & $\begin{array}{c}\text { BRS } \\
\text { Curinga }\end{array}$ & $\begin{array}{c}\text { BRS } \\
\text { Monarca }\end{array}$ & \\
\hline $\bar{x}$ & $94.26^{a}$ & $93.52^{a b}$ & $92.62^{b c}$ & $91.81^{c}$ & $90.61^{d}$ & 0,93 \\
\hline CV (\%) & 2.07 & 1.74 & 2.18 & 1.47 & 1.55 & - \\
\hline
\end{tabular}

$\bar{X}=$ media aritmética; $C V(\%)=$ coeficiente de variación.

- No determinado.

Líneas con letras iguales en las medias no difieren entre si por el teste de Tukey $(p>0.05)$.

TABLA 4 - Contenido de amilosa $\left(\right.$ dag $\left.\mathrm{kg}^{-1}\right)$ de cultivares de arroz de tierras altas sometidos a diferentes

\begin{tabular}{|c|c|c|c|c|c|c|}
\hline Tempo & $0 \mathrm{~min}$ & $10 \mathrm{~min}$ & $20 \mathrm{~min}$ & $30 \mathrm{~min}$ & $40 \mathrm{~min}$ & DMS \\
\hline $\bar{x}$ & $25.72^{a}$ & $24.62^{b}$ & $23.95^{c}$ & $23.05^{d}$ & $22.53^{e}$ & 0,48 \\
\hline CV (\%) & 3.81 & 3.97 & 3.22 & 4.30 & 4.26 & \\
\hline Cultivar & BRS Primavera & BRS Pepita & BRS Sertaneja & BRS Curinga & BRS Monarca & \\
\hline $\bar{x}$ & $25.19^{a}$ & $24.45^{b}$ & $24.05^{c}$ & $23.33^{d}$ & $22.82^{\mathrm{e}}$ & 0,39 \\
\hline CV (\%) & 4.94 & 4.77 & 4.90 & 5.99 & 5.09 & \\
\hline
\end{tabular}

\footnotetext{
$\bar{X}$ = media aritmética; CV $(\%)$ = coeficiente de variación. Líneas con letras iguales en las medias no difieren entre si por el teste de Tukey $(p>0.05)$.
} 
ASCHERI , D.P.R. et al. Calidad del arroz de tierras altas...

Los valores de almidón fueron superiores a los reportados por Pinkrová et al. (2003), que variaron de 84.4 a 87.7 dag $\mathrm{kg}^{-1}$, entretanto, los valores de amilosa se encontraron dentro del rango de valores obtenidos por González et al. (2004) que variaron de 18.0 a 26.7 dag $\mathrm{kg}^{-1}$, sin embargo inferior a los de Noosuk et al. (2003) para amilosa de arroz comercial (39.48 dag kg-1).

Entre los cultivares estudiados se detectaron mayores valores de almidón y de amilosa en BRS Primavera seguido, en orden descendente, por los cultivares BRS Pepita, BRS Sertaneja, BRS Curinga y BRS Monarca, en todo los tiempos de cocción (Tablas 3 y 4 , respectivamente).

Cabe resaltar que el proceso de cocción no aumenta ni disminuye la composición química del arroz, sin embargo, juega un papel muy importante en la liberación de sus componentes, una vez que estos están fuertemente ligados entre si formando estructuras complejas que mismo cocidos continúan parcialmente manteniendo esa estructura como es el caso del arroz cocido. En el presente estudio, se ha observado que con el aumento del factor tiempo aumentó el contenido del almidón y disminuyó el de amilosa, significando que el tiempo de cocción es un parámetro importante a ser considerado en la determinación de estos componentes y se llegó a la conclusión que mayor será la disponibilidad del contenido del almidón y menor el de amilosa cuanto mayor es el tiempo utilizado para la cocción de los granos de arroz de los diferentes cultivares estudiados. Boas et al. (2001) en sus estudios también demostraron que así como el tipo de extracción el factor tiempo es predominantemente importante en la determinación de azúcares totales, reductores y no reductores.

De acuerdo con Vitti (1966) y Bassinello et al. (2004), la absorción de agua, entre otros parámetros que determinan la calidad del arroz beneficiado durante la cocción, está directamente relacionada con la proporción de amilosa/ amilopectina del almidón. En el presente trabajo se verificó aumento de la absorción de agua de los granos de arroz con el aumento del tiempo de cocción con una tendencia a un determinado patamar entre los 30 a $40 \mathrm{~min}$, a partir del excedente de ese tiempo disminuye la capacidad de absorción de agua de los granos de arroz (Figura 1).

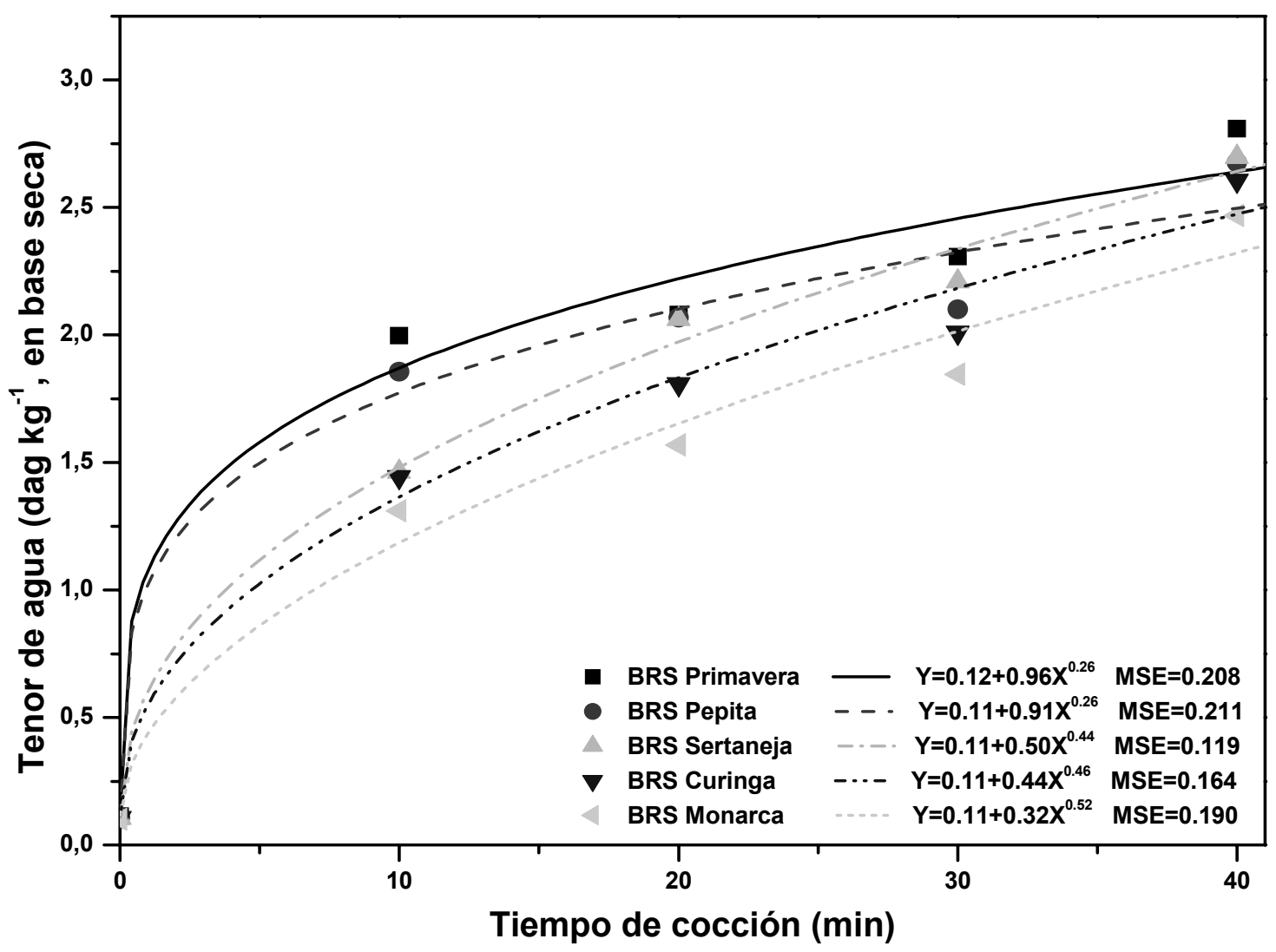

FIGURA 1 - Tenor de agua de cultivares de arroz de tierras altas en función del tiempo de cocción. MSE = cuadrado medio del error. 
ASCHERI , D.P.R. et al. Calidad del arroz de tierras altas...

Al confrontar valores medios del almidón y de la amilosa con los valores medios del contenido de agua a partir de los 10 min de cocción se verificó correlación linear positiva y correlación linear negativa para los pares almidón $x$ agua $(r \geq 0,959)$ e amilosa $x$ agua $(r \geq 0,860)$, respectivamente, en todos los cultivares de arroz estudiados (Tabla 5), indicando que cuanto mayor la disponibilidad del contenido de almidón y menor el contenido de amilosa mayor es la absorción de agua de los granos de arroz.

TABLA 5 - Correlación entre los contenidos de Al x Hu y Am x Hu de los granos de diferentes cultivares de arroz de tierras altas, sometidos a diferentes tiempos de cocción.

\begin{tabular}{lcc}
\hline \multirow{2}{*}{ Cultivar } & \multicolumn{2}{c}{ Correlación linear } \\
\cline { 2 - 3 } & Al $\times \mathrm{Hu}$ & $\mathrm{Am} \times \mathrm{Hu}$ \\
\hline BRS Primavera & 0,978 & $-0,988$ \\
BRS Pepita & 0,993 & $-0,941$ \\
BRS Sertaneja & 0,965 & $-0,990$ \\
BRS Curinga & 0,959 & $-0,978$ \\
BRS Monarca & 0,996 & $-0,860$ \\
\hline
\end{tabular}

$\mathrm{Al}=$ contenido de almidón; $\mathrm{Hu}=$ contenido de agua; $\mathrm{Am}=$ contenido de amilosa .

Los perfiles de viscosidad de pasta de las muestras crudas (Figura 2) muestran las modificaciones ocurridas en el almidón debido al efecto de los factores aplicados. Estas modificaciones dependen de la interacción $\mathrm{C} \times \mathrm{T}$ de acuerdo con el análisis de varianza de la Tabla 6 , aplicada al nivel de $1 \%$ de probabilidad. La viscosidad máxima fue alcanzada a $95{ }^{\circ} \mathrm{C} y$, de acuerdo con la prueba de Tukey $(p<0.05)$ (los resultados pueden ser observados en la misma Figura 2) los valores de Vmáx de los cultivares BRS Primavera (0.806 Pa s), BRS Monarca (0.870 Pa s) y BRS Sertaneja $(0.846 \mathrm{~Pa} \quad \mathrm{~s})$ fueron estadísticamente iguales y presentaron los menores valores, en cuanto que los cultivares BRS Pepita (1.279 Pa s) y BRS Curinga (1.192 Pa s) difirieron de los demás pero fueron estadísticamente iguales entre sí $(P>0.05)$. La ocurrencia de la retrogradación fue detectada entre 84 a $87{ }^{\circ} \mathrm{C}$. Estadísticamente fueron diferentes entre los granos de arroz crudos, con mayor tendencia a la retrogradación para los cultivares BRS Pepita (2.769 Pa s) y BRS Curinga (2.447 Pa s) seguidos, en orden decreciente, para los cultivares BRS Monarca (1.463 Pa s), BRS Sertaneja (1.449 Pa s) y BRS Primavera (1.374 Pa s).

TABLA 6 - Análisis de varianza de la viscosidad máxima (Vmáx) e ocurrencia de retrogradación (Sb) de diferentes cultivares de arroz de tierras altas sometidos a diferentes tiempos de cocción.

\begin{tabular}{lccrrr}
\hline & & \multicolumn{2}{c}{ Cuadrado medio } & \multicolumn{2}{c}{ Prueba F } \\
\cline { 3 - 6 } Fuente de variación & G.I. & Vmáx & Sb & Vmáx & Sb \\
\hline Cultivar (C) & 4 & 0.119 & 0.401 & $5947.2^{* * *}$ & $1030.2^{* * *}$ \\
Tiempo (T) & 4 & 1.858 & 8.729 & $93090.7^{* * *}$ & $22411.7^{* * *}$ \\
C x T & 16 & 0.031 & 0.245 & $1543.4^{* * *}$ & $630.3^{* * *}$ \\
\hline
\end{tabular}

G.I. = grados de libertad; ${ }^{* * *}$ Muy significativo al nivel de $0.1 \%(p<0.001) ;-$ No determinado. 
ASCHERI , D.P.R. et al. Calidad del arroz de tierras altas...

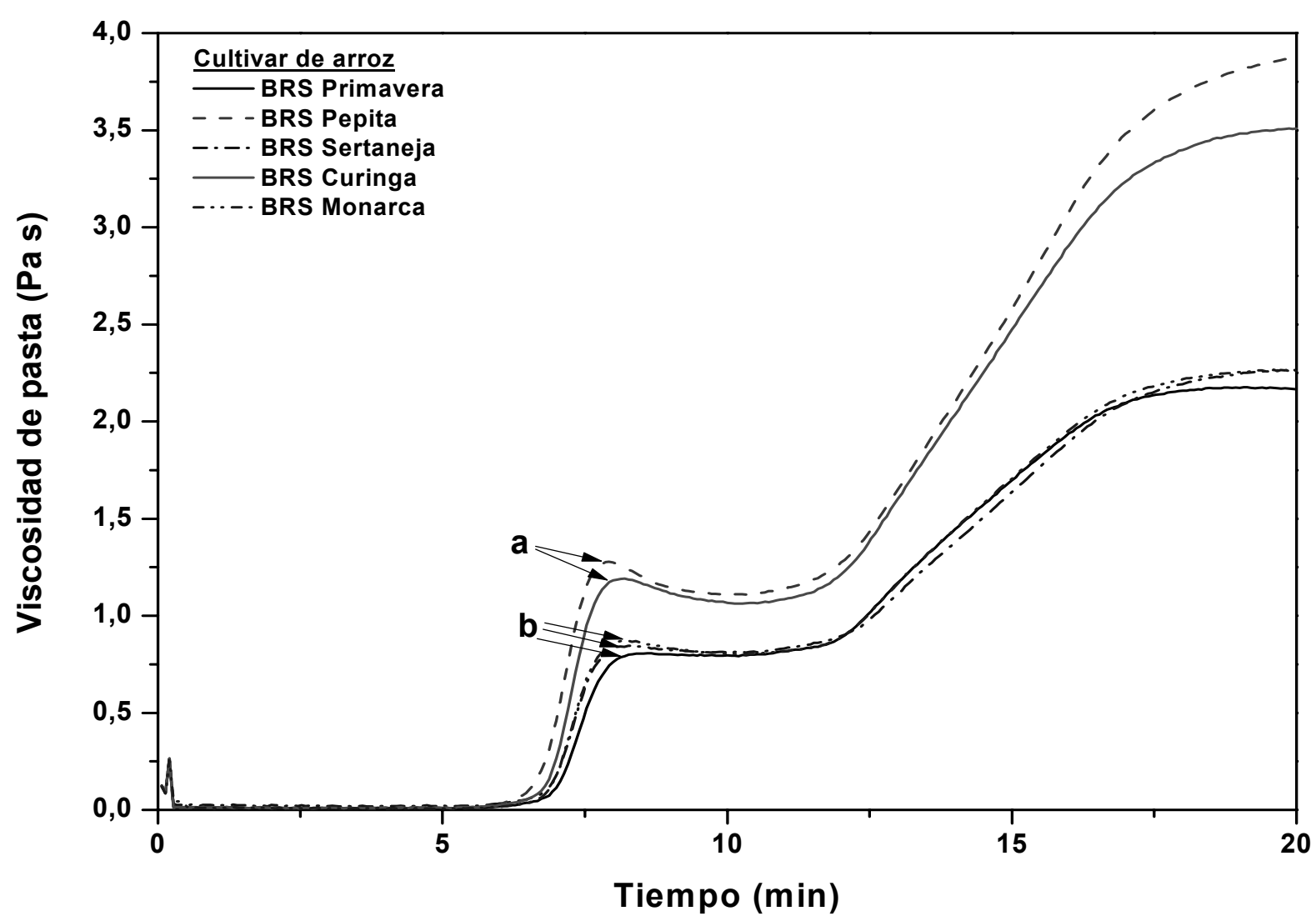

FIGURA 2 - Perfiles de la viscosidad de pasta de los granos crudos de diferentes cultivares de arroz de tierras altas. Curvas con letras iguales en las viscosidades máximas no difieren entre si por el teste de Tukey $(p>0.05)$.

En la Figura 3 se observa el efecto causado por los factores C e T $(p<0.01)$ en los granos de arroz cocidos. El tiempo de cocción alteró significativamente el perfil de viscosidad de la Figura 2, reduciendo la viscosidad en tiempos más prolongados. Similares efectos fueron observados por otros autores en almidón de granos de arroz irradiados por microondas (Pinkrová et al., 2003) e irradiados con rayos gamma (Bao et al., 2005; Yu \& Wang, 2007; Zanão et al., 2009) que observaron que el aumento de la exposición del arroz a la irradiación causaba disminución de las propiedades de las pastas.

La interacción C x TC de esos parámetros de viscosidad se muestran en la Figura 4. Las curvas son semejantes, disminuyen asintoticamente con el tiempo de cocción. El cultivar BRS Curinga ofrece menor resistencia a la gelatinización (Figura 4a) y retrogradación (Figura 4b), presentando los mayores valores de Vmáx y de $\mathrm{Sb}$, indicando que los granos cocidos de este cultivar pueden absorber mayor cantidad de agua cuando calientes y ser más pegajosos cuando fríos. Por el contrario, los demás cultivares (excepto el arroz crudo del cultivar BRS Pepita) presentan alta resistencia, sugiriendo que estos granos de arroz podrán presentar granos cocidos sueltos y suaves, es decir, pocos pegajosos. Estos resultados refuerzan los trabajos realizados por el programa de mejoramiento de arroz de tierras altas de la Embrapa Arroz e Feijão en que utilizaron el cultivar BRS Pepita como muestra de comparación en los ensayos de cocción, para la evaluación de linajes preemisores, cuyos granos se presentan sueltos en el cocimiento, mismo cuando el producto es recién cosechado (Castro et al., 1999; Fonseca et al., 2004). 
ASCHERI , D.P.R. et al. Calidad del arroz de tierras altas...
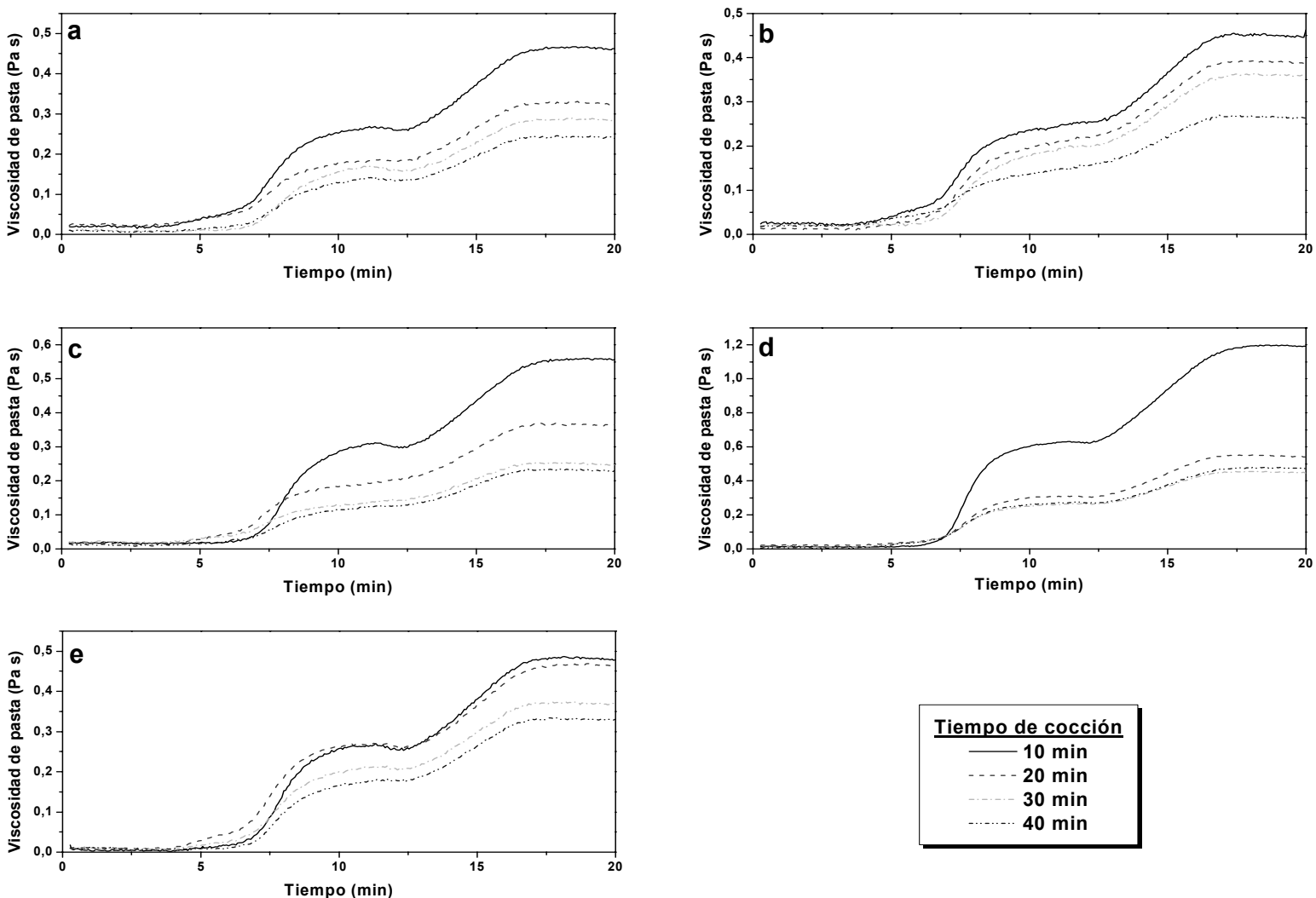

FIGURA 3 - Perfiles de la viscosidad de pasta de los granos de arroz cocidos en diferentes tiempos de cocción de los diferentes cultivares de tierras altas: a) BRS Primavera, b) BRS Pepita, c) BRS Sertaneja,

d) BRS Curinga y e) BRS Monarca.

El ensayo de cocción fue hecho simulando el preparado doméstico del arroz. A través de ese ensayo, se puede evaluar la calidad de cada muestra en relación a la textura, a la pegajosidad, al rendimiento y al tiempo de cocción. Estos parámetros pueden ser vistos en la Tabla 7 . En relación al atributo de pegajosidad, apenas el cultivar BRS Primavera presentó un aspecto de granos cocidos sueltos. Este cultivar es considerado patrón en el sistema de cultivo de tierras altas. En relación al atributo de textura, no hubo diferencia entre las muestras, pues, se presentaron con característica de arroz cocido duro. Algunos factores pueden haber influenciado en esos resultados, como por ejemplo, la manera de analizar la textura de la muestra, que es realizada cuando esta ya se encuentra fría y el proceso de retrogradación del almidón instalado en la misma. En relación al rendimiento y al tiempo de cocción, el cultivar BRS Primavera fue el que más rindió y el que se cocinó en menos tiempo. De acuerdo con Castro et al. (2003), granos de arroz con temperatura de gelatinización intermediaria y baja, como el cultivar BRS Primavera, por ejemplo, requieren menos tiempo de cocción.

TABLA 7 - Calidad de diferentes cultivares de arroz de tierras altas, en relación a la pegajosidad, textura, rendimiento y tiempo de cocción.

\begin{tabular}{lcccc}
\hline \multicolumn{1}{c}{ Cultivar } & Pegajosidad & Textura & Rendimiento (\%) & Tiempo (min) \\
\hline BRS Primavera & S & D & 250 & 21 \\
BRS Pepita & LP & D & 225 & 22 \\
BRS Sertaneja & LP & D & 225 & 23 \\
BRS Curinga & P & D & 200 & 23 \\
BRS Monarca & LP & D & 225 & 22 \\
\hline
\end{tabular}

S - Suelto; LP - Ligeramente pegajoso; P - Pegajoso, D-Duro. 
ASCHERI , D.P.R. et al. Calidad del arroz de tierras altas...
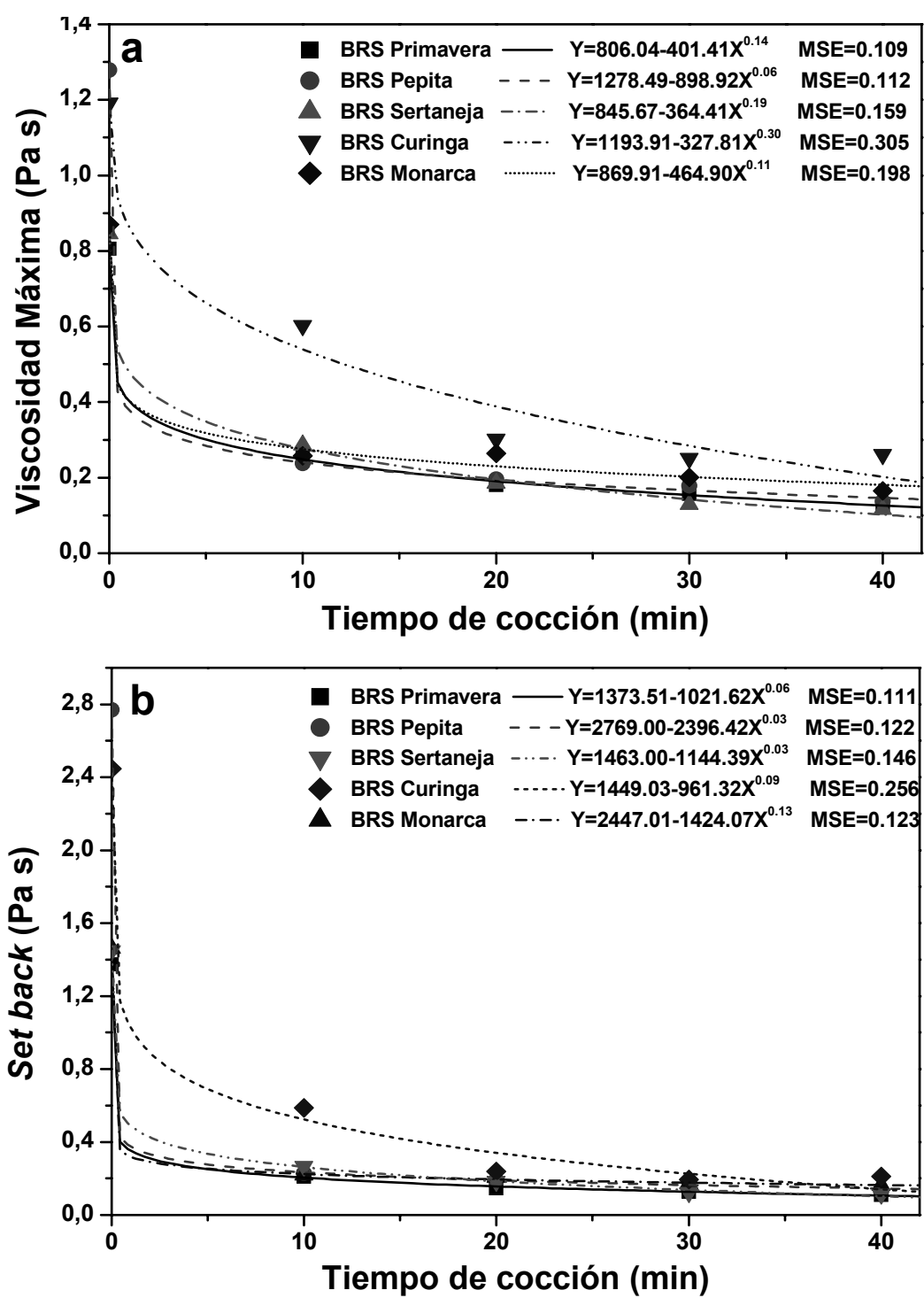

FIGURA 4 - Variación de la viscosidad de pasta de harinas de diferentes cultivares de arroz de tierras altas en función del tiempo de cocción: a) viscosidad máxima y b) tendencia a la retrogradación (set back). MSE = cuadrado medio del error

Según Ferreira et al. (2005), gran parte de América Latina prefiere arroz de baja pegajosidad después de la cocción, de forma que los granos queden sueltos. Esta propiedad está relacionada al contenido de amilosa, siendo así los cultivares de arroz pueden ser clasificadas como bajo contenido

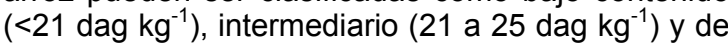
alto contenido (>25 dag $\mathrm{kg}^{-1}$ ). Alto contenido de amilosa normalmente resulta en granos secos y sueltos que pueden endurecer después de enfriados. Un bajo contenido resulta en granos suaves, acuosos y pegajosos durante la cocción. Considerando esta clasificación, los cultivares con contenido intermediario son los preferidos, pues, presentan granos secos y sueltos después de la cocción y se mantiene suave en el recalentamiento.

A pesar de los cultivares estudiados presentaren contenido de amilosa dentro del rango intermediario, al compararse con los resultados obtenidos por medio de los ensayos de cocción (Tabla 7) se observa que otros factores así como la amilosa influencian en la calidad del arroz. La amilosa se relacionó linealmente con la viscosidad máxima y con la tendencia a la retrogradación (Tabla 5). Cuanto menor es el contenido de la amilosa menores son los valores de la viscosidad máxima y de la tendencia a la retrogradación.

\section{CONCLUSIONES}

Los factores cultivar y tiempo de cocción afectan los contenidos de almidón y amilosa. Los contenidos de almidón varió de 90.61 a 94.26 dag $\mathrm{kg}^{-1}$ y el contenido de amilosa varió de 22.82 a 25.19 dag $\mathrm{kg}^{-1}$. El cultivar BRS Primavera presentó mayores valores de estos constituyentes seguidos 
ASCHERI , D.P.R. et al. Calidad del arroz de tierras altas...

por los cultivares BRS Pepita, BRS Sertaneja, BRS Curinga y BRS Monarca. La disponibilidad de determinación del almidón es mayor y de la amilosa es menor cuanto mayor es el tiempo de cocción.

El contenido del agua varía de acuerdo con la interacción cultivar $x$ tiempo de cocción. El tiempo límite de absorción de agua de los granos de arroz está entre 30 y 40 min de cocción, siendo la disponibilidad del contenido de almidón el responsable por la absorción y presenta correlación lineal positiva con en contenido de agua de los granos de arroz de los diferentes cultivares estudiados. La amilosa presentó correlación lineal negativa con la presencia del contenido del agua.

La viscosidad máxima y la tendencia a la retrogradación dependen de la interacción cultivar $x$ tiempo de cocción, cuanto mayor es el tiempo de cocción del arroz menores son los valores de estas propiedades de pasta de los granos de arroz de las cultivares de tierras altas.

Los granos de arroz de los cultivares BRS Pepita y BRS Curinga pueden absorber mayor cantidad de agua cuando calientes y pueden presentarse más pegajosos cuando fríos y los cultivares BRS Primavera, BRS Sertaneja y BRS Monarca pueden absorber menor cantidad de agua cuando calientes y sus granos pueden presentarse secos y sueltos y endurecer después de enfriados.

\section{AGRADECIMIENTO Y CONFLICTOS DE INTERÉS}

A la Embrapa Arroz e Feijão y a la Universidad Estatal de Goiás por las prácticas preprofesionales y realización de las análisis.

El presente trabajo no presenta conflictos de interés que sean del conocimiento de los autores.

\section{REFERENCIAS}

1. AMERICAN ASSOCIATION OF CEREAL CHEMISTS. Approved methods of the American Association of Cereal Chemists. 7. ed. Saint Paul: AACC, 1969. v. 2.

2. ÁVILA, M. Evaluación de la calidad culinaria del arroz. Una herramienta para el mejoramiento genético. DANAC Boletín Informativo, v. 7, n. 1, p. 1-2, 2001.

3. BAO, J.; AO, Z. H.; JANE, J. L. Characterization of physical properties of flour and starch obtained from gammairradiated white rice. Starch/Stärke, v. 57, n. 10, p. 480-487, 2005.

4. BASSINELLO, P. Z.; ROCHA, M. S.; COBUCCI, R. M. A. Avaliação de diferentes métodos de cocção de arroz de terras altas para teste sensorial. Santo Antônio de Goiás: Embrapa, 2004. 8 p. (Comunicado técnico, 84).

5. BOAS, B. M. V. et al. Seleção de extratores e tempo de extração para determinação de açúcares em café torrado. Ciência e Agrotecnologia, v. 25, n. 5, p. 1169-1173, 2001.

6. CASTRO, E. M.; FERREIRA, C. M.; MORAIS, O. P. Qualidade de grãos e competitividade do arroz de terras altas. In: CONGRESSO DA CADEIA PRODUTIVA DE ARROZ, 1., REUNIÃO NACIONAL DE PESQUISA DE ARROZ, 7. 2002, Florianópolis. Anais... Santo Antônio de Goiás: Embrapa Arroz e Feijão, 2003. v. 2. p. 201-214.

7. CASTRO, E. M. et al. Qualidade de grãos em arroz. Santo Antônio de Goiás: Embrapa Arroz e Feijão, 1999.30 p. (Circular Técnica, 34)

8. CEREDA, M. P.; DAIUTO, E. R.; VILPOUX, O. Metodologia de determinação de amido digestão ácida em microondas. Associação Brasileira dos Produtores de Amido de Mandioca, v. 2, n. 8, s/p, 2004. Disponible en: <http://www.abam.com.br/revista/revista8/metodologia.php>. Acceso en: 11 out. 2009.

9. FOOD AND AGRICULTURE ORGANIZATION OF THE UNITED NATIONS (FAO). Año internacional del arroz: todo sobre el arroz. Roma. 2004. Disponible en: <http://www.fao.org/rice2004/es/aboutrice.htm>. Acceso en: 16 set. 2009.

10. FERREIRA, C. M., et al. Qualidade do arroz no Brasil: evolução e padronização. Brasília: Embrapa Arroz e Feijão, 2005. 61p

11. FONSECA, J. R. et al. Recomendações de cultivares de arroz de terras altas para o Estado do Tocantins. Santo Antônio de Goiás: Embrapa Arroz e Feijão, 2004. 7 p. (Circular Técnica, 66).

12. GOMES, F. P. Curso de estatística experimental. 12. ed. São Paulo: Nobel, 1987. 467 p.

13. GONZÁLEZ, R. J.; LIVORE, A.; PONS, B. Physico-chemical and cooking characteristics of some rice varieties. Brazilian Archives of Biology and Technology, v. 47, n. 1, p. 71-76, 2004.

14. JULIANO, B. O. Criteria and tests for rice grain qualities. In: JULIANO, B. O. (Ed.). Rice: chemistry and technology. 2. ed. Saint Paul: American Association Cereal Chemistry, 1985. p. 443-524.

15. KENNEDY, G.; BURLINGAME, B. Analysis of food composition data on rice from a plant genetic resources perspective. Food Chemistry, v. 80, n. 4, p. 589-596, 2003.

16. MARTINEZ, C.; CUEVAS, F. Evaluación de la calidad culinaria y molinera del arroz: guía de estudio para ser usada como complemento de la unidad auditutorial sobre el mismo tema. 3. ed. Cali: Centro Internacional de Agricultura Tropical, 1989. $73 \mathrm{p}$.

17. NEWPORT SCIENTIFIC. Aplications manual for the Rapid Visco Analyser. Austrália: Instrument Support Group, 1998. $123 \mathrm{p}$.

18. NOOSUK, P. et al. Structure-viscosity relationships for thai rice starches. Starch/Stärke, v. 55 , n. 8 , p. $337-344$, 2003.

19. PINKROVÁ, J. et al. Changes of starch during microwave treatment of rice. Czech Journal of Food Science, v. 21, n. 5, p. 176-184, 2003.

20. SCHOLZ, M. B S S : MAGRI, T. B. Tempo de cozimento de cultivares e linhagens de arroz irrigado. In: CONGRESSO DA CADEIA PRODUTIVA DE ARROZ, 1.; REUNIÃO NACIONAL DE PESQUISA DE ARROZ, 7., 2002, Florianópolis. Anais... Santo Antônio de Goiás: Embrapa Arroz e Feijão, 2003. v. 1, p. 51-53.

21. STATSOFT. Electronic Statistics Program. Tulsa: StatSoft, 2007. 1 CD-ROM.

22. VITTI, P. A. Viscosidade do amido e sua importância nos alimentos. Boletim do Centro Tropical de Pesquisa e Tecnologia de Alimentos, n. 8, p. 69-83, 1966. 
ASCHERI , D.P.R. et al. Calidad del arroz de tierras altas...

23. WANG, S. H. et al. Estudo das propriedades reológicas e sensoriais após reconstituição dos mingaus desidratados de arroz e soja. Ciência Tecnologia de Alimentos, v. 20, n. 1, p. 68-73, 2000.

24. YU, Y.; WANG, J. Effect of $y$-ray irradiation on starch granule structure and physicochemical properties of rice. Food Research International, v. 40, n. 2, p. 297-303, 2007.

25. ZANÃO, C. F. P. et al. Efeito da irradiação gama nas características físico-químicas e sensoriais do arroz (Oryza sativa L.) e no desenvolvimento de Sitophilus oryzae L. Ciência e Tecnologia de Alimentos, v. 29, n. 1, p. 46-55, 2009.

Recebido em: 26/02/2009

Aceito em: 23/10/2009 
